


\title{
Apropiaciones y resignificaciones de las "culturas negras": la práctica del candombe afrouruguayo en sectores juveniles blancos de Buenos Aires (Argentina) ${ }^{1}$
}

\author{
Eva Lamborghini ${ }^{2}$ \\ Universidad de Buenos Aires, Argentina \\ lamborghinieva@yahoo.com.ar \\ Recibido: 1. ${ }^{\circ}$ de febrero de 2016 \\ Aceptado: 22 de abril de 2016 \\ Disponible en línea: 20 de diciembre de 2016
}

El texto presentado corresponde a un artículo de reflexión teórica, cuyos argumentos tienen como base el trabajo de campo etnográfico realizado en el marco de la investigación doctoral de la autora (Lamborghini, 2015).

2 Doctora en Antropología, Universidad de Buenos Aires (UBA) (Argentina). Becaria postdoctoral, Consejo Nacional de Investigaciones Científicas y Técnicas (CONICET). Miembro investigadora, Grupo de Estudios Afrolatinoamericanos (GEALA), Instituto de Historia Argentina y Americana Dr. Emilio Ravignani, UBA (Buenos Aires, Argentina). 


\title{
Apropiaciones y resignificaciones de las "culturas negras": la práctica del candombe afrouruguayo en sectores juveniles blancos de Buenos Aires (Argentina) \\ Resumen
}

El artículo analiza la expansión de una manifestación cultural afrouruguaya, el candombe, en Argentina, un país que históricamente se ha pensado como "blanco-europeo". El candombe de origen uruguayo fue primero patrimonio de los inmigrantes afrouruguayos en Buenos Aires; posteriormente, trascendió la comunidad migrante que lo introdujo, fue enseñado y transmitido a distintos sectores sociales, y en la actualidad es parte de la cultura juvenil de cada vez más ciudades del país. Este trabajo se focaliza en sus apropiaciones y resignificaciones por parte de jóvenes de sectores medios y socialmente blancos, y aspira a contribuir a la problematización de procesos -escasamente estudiados-relacionados con el despliegue de "cultura negra" más allá de colectivos socialmente negros, señalando intersecciones entre perspectivas teóricas relativas al campo de los estudios afrolatinoamericanos, las culturas juveniles y los nuevos movimientos sociales. El texto muestra cómo las culturas afroamericanas, lejos de constituir patrimonios congelados, se encuentran en permanente construcción de significados y sentidos de interpelación diversos, en ocasiones mucho más allá de las poblaciones que las originaron.

Palabras clave: candombe afrouruguayo; Buenos Aires; culturas negras; culturas juveniles; movimientos sociales

\section{Re-Appropriation and Re-Signification of "Black Culture": Afro-Uruguayan Candombe Practice by Socially White Youth of Buenos Aires (Argentina)}

\begin{abstract}
The article analyzes the growth of Afro-Uruguayan candombe in a country -and, especially, a city, Buenos Aires - that has historically seen and considered itself as "White-European." The Uruguayan candombe was first cultural heritage of Afro-Uruguayan immigrants in Buenos Aires; later, this practice went beyond the migrant community, and it was taught and transmitted to various social sectors. It is currently part of the youth culture of an increasing number of cities in the country. The paper focuses on the re-appropriation and re-signification of Afro-Uruguayan candombe by young, middle-class, socially white Argentineans. It aims to understand the deployment of "black culture" beyond socially black groups - a scantly studied topic-, employing theoretical perspectives from "Afro-Latin American studies," "youth cultures," and "new social movements." The paper shows how Afro-American cultures, far from being frozen assets, are subject to permanent constructions of meaning and ways of interpellation that reach far beyond the populations that originated them
\end{abstract}

Keywords: Afrouruguayan candombe; Buenos Aires; black cultures; youth cultures; social movements

\section{Apropriações e ressignificações das "culturas negras": a prática do candomblé afro-uruguaiano em setores juvenis brancos de Buenos Aires (Argentina)}

\section{Resumo}

O artigo analisa a expansão de uma manifestação cultural afro-uruguaiana, o candomblé, na Argentina, um país que tem sido historicamente considerado como "branco-europeu". O candomblé de origem uruguaiana foi primeiramente herança dos imigrantes afro-uruguaianos em Buenos Aires; posteriormente ultrapassou a comunidade migrante que introduziu, foi ensinado e transmitido para diversos setores sociais, e hoje faz parte da cultura jovem de cada vez mais cidades do país. Este trabalho se concentra em suas apropriações e ressignificações por parte de jovens de setores médios e socialmente brancos e tem como objetivo contribuir para a problematização de processos - escassamente estudados- relacionados com a implantação de "cultura negra" para além de grupos sociais negros, apontando para intersecções entre perspectivas teóricas relativas ao campo de estudos afro-latino-americanos, culturas juvenis e novos movimentos sociais. O texto mostra como é que culturas afroamericanas, longe de serem ativos congelados, estão em permanente construção de significados e sentidos de interpelação variados, às vezes muito além das populações que lhes deram origem.

Palavras-chave: candomblé afro-uruguaiano; Buenos Aires; culturas negras; culturas juvenis; movimentos sociais 


\section{Introducción}

En Argentina, un país cuya identidad nacional se configuró bajo el ideal de "blanquedad" - y no de mestizaje como en otras naciones latinoamericanas- (Frigerio, 2006), la creciente valoración de la diversidad cultural, propia de contextos de apreciación multiculturalistas, ha favorecido la emergencia y el afianzamiento de procesos de reafricanización en la sociedad (Frigerio y Lamborghini, 2011b).

Entre estos, se destaca la formación de un movimiento social de afrodescendientes que luchan por su revisibilización y por el reconocimiento de sus aportes sociales y culturales a la nación. Así mismo, coadyuvada por los procesos de democratización de saberes y prácticas populares en general en las últimas décadas, la "cultura negra" (Frigerio, 2000; Hall, 1998/2003; Sansone, 2003) cobra cada vez mayor relieve. Este desarrollo se advierte no solo en algunos discursos y actividades apoyados por el Estado - como parte de las políticas de lo afro/descendiente- sino, sobre todo, en una movida cultural afro autónoma que crece y se vigoriza y que, como veremos, coincide con algunos de los cambios que se producen en nuestra escena cultural contemporánea.

En este artículo me propongo desarrollar aspectos teórico-reflexivos relacionados con la expansión del candombe de origen afrouruguayo $^{3}$ en Buenos Aires, un fenómeno que cobra creciente importancia en el desarrollo de nuevas formas de expresividad cultural -y también política- en el espacio público de esta ciudad - y de cada vez más ciudades del país-. La práctica de esta manifestación de "cultura popular negra" (Ferreira, 2013) ha ido conformando un campo

3 La performance del candombe es popular, colectiva y callejera. La música candombe surge de la interacción del conjunto de las células rítmicas de los tambores. Cada tamborilero/a toca un tambor, con la mano y/o con un palo, ya sea parados o caminando: "La orquesta de tambores (denominada cuerda o batea), multiplica en una formación en hileras y columnas, al grupo básico de tres tambores: menor, mediano y grande, de respectivas sonoridades aguda media y grave y, denominados 'chico', 'repique' y 'piano"' (Ferreira, 2008, p. 98). Los/las músicos y bailarinas/es se agrupan en comparsas que se reúnen a ensayar y preparan presentaciones, salidas por la calle y desfiles. Las comparsas son acompañadas por una audiencia de personas que participan bailando y siguiendo el desfile o ensayo. Tanto el toque de los tambores como la danza del candombe están relacionados con principios culturales afroamericanos: la polirritmia, la policentralidad energética, la forma circular del movimiento, el sentido de un todo y la intensificación por la repetición (Ferreira, 2008, p. 98). 
(Bourdieu, 2011) ${ }^{4}$ sumamente dinámico y en continuo crecimiento desde su transnacionalización y relocalización por una primera generación de inmigrantes afrouruguayos en la década de $1980^{5}$, que trascendió luego la comunidad migrante de origen y fue incorporada por distintos sectores sociales. Dentro de este desarrollo, mis argumentos se centrarán en dimensiones relativas a su práctica - no a su consumo- por sectores juveniles socialmente "blancos" que se suman pujantemente al candombe en la última década. A lo largo de estos años, tales sectores han organizado actividades aglutinadoras y colectivos candomberos particulares, construyendo caminos alternativos que corren paralelos al circuito del candombe mayor, en el que también convergen. El trazado de una movida candombera con rasgos singulares armoniza con algunas de las caracteristicas de cierto espíritu de época correspondiente a la socialización generacional de estos tomborileros/as y bailarinas. Estos rasgos, a su vez, resultan en innovaciones respecto de un modelo más tradicional afrouruguayo. Con este trasfondo, los sectores juveniles que enfocamos, menos legitimados en este "espacio negro" (Sansone, 2003) ${ }^{6}$ local, tensionan la idea de propiedad con la de apropiación de esta expresión cultural.

Sostener que existen distintas construcciones del candombe en Buenos Aires - $-\mathrm{y}$ en Argentina en sentido más extenso- nos aleja de la idea de una misma manifestación cultural compartida por todos a-conflictivamente. Sin embargo, en tanto que en el campo candombero local se producen convergencias en varios eventos (Llamadas -desfiles de tambores-, reuniones, toques, fiestas u otras iniciativas) y relaciones solidarias que exceden a los grupos de pertenencia y más próximos, lejos estoy de plantear divisiones o abismos insalvables; tal vez los varios caminos confluyan cada vez más. En cambio, es

4 Esta noción resulta adecuada para pensar cómo los sujetos tejen relaciones de acuerdo a su posesión de desiguales capitales sociales y simbólicos, disputando significados y la definición de lo legítimo y lo ilegítimo.

5 Aludo más precisamente al proceso de transnacionalización desde abajo (Frigerio, 2013) del candombe desde Montevideo a Buenos Aires. El amplio proceso de difusión del candombe en el escenario local está relacionado —aunque no determinado - con esta forma de llegada, y no con su circulación como un producto simbólico trasladado y/o ofrecido como mercancía para consumo, como se advierte críticamente que sucede con la mercantilización globalizada de géneros tradicionales afroamericanos (Carvalho, 2002).

6 Considero que es posible pensar el campo del candombe como un "espacio negro", extrapolando la categoría del análisis de Sansone (2003) sobre el contexto bahiano-brasilero. El autor nos dice que en "espacios negros" como el candomblé y determinadas actividades recreativas como la capoeira, la samba, las asociaciones de carnaval, etc., la identificación como negro es considerada incluso una ventaja, a diferencia de otras áreas tanto duras como blandas del racismo y/o de las relaciones raciales (Sansone, 2003, pp. 52, 53 y 206). 
mi intención dar cuenta de estas construcciones diferentes del candombe, aspirando específicamente a contribuir a la problematización de fenómenos - escasamente abordados- relacionados con el despliegue de "culturas negras" por fuera de los límites étnico-raciales tradicionales, es decir; con prácticas culturales negras más allá de identidades negras.

Este problema, que en principio tiene que ver con que los secretos internos y las reglas étnicas de las artes performáticas afroamericanas pueden transmitirse y aprenderse (Corti, 2011, p. 126, siguiendo a Gilroy, 1993), nos remite en términos teóricos más amplios a los llamados de atención sobre la disociación entre cultura - raza - identidad - etnicidad - negras- (Frigerio, 2000; Sansone, 2003) ${ }^{7}$. En esta línea, ponemos de relieve la necesidad de abordar procesos socioculturales de apropiación de prácticas culturales afroamericanas de manera más compleja, ya que, así como la mayor parte de los análisis actuales sobre "cultura negra" examinan procesos identitarios y/o de la movilización politico/cultural afrodescendiente, la bibliografia que toma la participación blanca en la/s cultura/s negra/s suele abordar procesos de apropiación con eje en relaciones de poder ejercidas desde sectores blancos dominantes, entrando en juego nociones como robo, banalización, mercantilización, etc. (Carvalho, 2002; Sheriff, 1999).

Teniendo como base este marco general de reflexión, en este artículo indicaremos cómo en el caso del candombe afrouruguayo en Buenos Aires -y en Argentina en sentido más extenso- se destaca la incumbencia de temas del campo de los estudios afrolatinoamericanos (Guzmán y Geler, 2013; Restrepo, 2013; Wade, 2006), pero también su cualidad de vértice de otros que lo exceden, señalando sus conexiones con las transformaciones sociales resultantes de la crisis neoliberal de fines de la década del noventa y de 2001 (Wortman, 2009, 2010); con las formas de interfase entre politica y cultura que desde entonces se destacan; los nuevos movimientos sociales (Goldman, 2007) que se expresan colectivamente en el espacio público (Svampa,

Es siguiendo esta perspectiva de trabajo que considero que el estudio remite a un enfoque no de negritud sin etnicidad (Sansone, 2003), ni de identidad negra sin cultura negra (Frigerio, 2000), sino de cultura negra sin identidad negra, o bien, como indiqué previamente, con la posibilidad de ubicarse más allá de la identidad negra (Rinaudo, 2010). 
2008, 2011) $)^{8}$ y con los lenguajes y modos de hacer compartidos con las culturas juveniles (Reguillo, 2000/2012), poniendo en evidencia la multidimensionalidad necesaria para su abordaje.

\section{Candombe afrouruguayo en Buenos Aires: un breve racconto}

El candombe en la región del Río de la Plata nos remite a la historia de organización y recreación comunitaria de las africanas y africanos esclavizadas/os, y luego de sus descendientes, en la época colonial y en los contextos de las naciones independientes. Como parte de las prácticas musicales y dancísticas que reflejan un legado de origen africano, los candombes fueron adaptados al contexto social y cultural del momento, en diálogo con las estructuras de poder y siendo objeto de negociaciones, prohibiciones y estigmatizaciones. Cuando hacia las últimas décadas del siglo XIX Uruguay y Argentina, como otros países de Latinoamérica, se encaminaron hacia la "modernidad" y se consolidaron como estados-nación, la población afrodescendiente - la "gente de color" - se vio implicada en procesos de "regeneración" de sus antiguas costumbres, negociando así su acceso a las ciudadanías en las repúblicas modernas. Sin embargo, las políticas del carnaval, el lugar de "lo negro" en general y del candombe en particular, tomaron caminos distintos -inversos- en las ciudades capitales de cada margen del Plata. Un punto saliente cuando se piensan los diferentes desarrollos del candombe en Montevideo y Buenos Aires es la visibilidad/invisibilidad y el crecimiento/ocaso de ambas modalidades en los espacios públicos urbanos respectivos en épocas de conformación nacional-estatal (Andrews, 2007; Geler, 2011). De acuerdo

A fines de 2001 se produjeron en Argentina una serie de sucesos denominados la "crisis de 2001". En un contexto de larga recesión económica, de una serie de políticas económicas y sociales antipopulares y avasallantes de derechos sociales que redundaron en una crisis de representatividad política, resumida en el lema "que se vayan todos" (los políticos), el 19 de diciembre de 2001 se produjo una rebelión policlasista — sectores medios y populares aunados bajo un "cacerolazo"-, que causó la renuncia del presidente de la nación. Las jornadas de este y del día siguiente fueron reprimidas por el gobierno, dejando un saldo de 39 manifestantes asesinados. Siguiendo a Svampa (2011), las jornadas del 19 y el 20 de diciembre de 2001 abrieron un nuevo ciclo de acción colectiva. Si bien con antecedentes en la década de 1990, será a partir de diciembre de 2001 que el país ingrese en un periodo marcado por intensas movilizaciones sociales, proclives a la acción directa y a la auto-organización desde abajo, potenciando la presencia de estos grupos en el espacio público. 
con la configuración de distintas formaciones nacionales de alteridad de organización de las diferencias y la desigualdad (Briones, 2005; Segato, 1998), mientras que el candombe argentino fue sufriendo un proceso de repliegue al ámbito de lo privado de las familias afroargentinas -experimentando un reciente retorno público (Cirio, 2007)-, en Uruguay se popularizó masivamente (Ferreira, 2013), y fue también trascendiendo las fronteras nacionales ${ }^{9}$.

Desaparecidas las antiguas naciones africanas que poblaron el barrio de San Telmo y el contiguo Montserrat - los barrios que contaron en el pasado con una importante población negra-, la "vuelta del tambor" en Buenos Aires se produjo a comienzos de la década de 1980, cuando inmigrantes afrouruguayos comenzaron a reproducir en la Plaza Dorrego de San Telmo las Llamadas/salidas de tambores que se realizan en Montevideo en varios barrios de la ciudad en determinados días feriados a lo largo del año, reconstituyendo así lazos en contexto de migración con un fuerte sentido de nacionalidad - uruguaya- $\left(\right.$ Parody, 2014) ${ }^{10}$. A partir de 1990, referentes de una segunda generación de inmigrantes afrouruguayos comenzaron a enseñar y difundir su arte a otros sectores sociales, realizando conexiones explícitas entre su presencia actual y el pasado negro porteño y reivindicando los aportes de los afroargentinos a la cultura del país ${ }^{11}$. A partir de 2000, la disolución - unos años antes- de la primera comparsa de estilo montevideano en Argentina -denominada Kalakan-Güé ${ }^{12}$ - dio lugar a una primera generación de comparsas

9 Aunque presente ya en varios países, la expansión del candombe fuera de las fronteras nacionales uruguayas ha merecido hasta el momento solo estudios en Argentina.

10 En este trabajo no indago la llegada del candombe relacionada con la recepción de material discográfico y/o de los shows de músicos uruguayos. Sin duda, estos procesos, así como las performances de músicos de la primera generación de migrantes afrouruguayos en circuitos de músicos profesionales, nutrieron durante tres décadas a músicos y percusionistas argentinos (Parody, 2014, p. 140) y no están desvinculados de la prolífica difusión de la práctica del candombe callejero actual. Esto es aun más notorio en los contextos provinciales en que la práctica del candombe llegó inicialmente mediante músicos locales atraídos por el género - y/o sus conexiones con Uruguay- y no por inmigrantes afrouruguayos como sí sucedió en la capital (Broguet, Picech y Rodríguez, 2013).

11 Se trata, principalmente, de los emprendimientos del Grupo Cultural Afro, integrado por los hermanos Acosta Martínez y Bonga.

12 En 1996 uno de los fundadores del Grupo Cultural Afro, José Delfín Acosta Martínez, fue asesinado brutalmente en un episodio de racismo policial. Su hermano, Ángel Acosta Martínez, redobló sus labores docentes en centros culturales de la ciudad y a partir de 1997 se dedicó a la organización de una comparsa que reivindicara la memoria de su hermano y, a la vez, la de los negros argentinos. El 13 de diciembre de 1998 sus esfuerzos se cristalizaron en un gran desfile por el barrio de San Telmo hasta la Plaza de Mayo denominado Homenaje a la Memoria, que tuvo a la comparsa KalakanGüé, formada casi íntegramente por alumnos argentinos de Ángel Acosta, como protagonista principal. Aunque al tiempo Kalakan-Güé se disolvió, tuvo un efecto multiplicador ya que muchos de sus integrantes formaron sus propias 
porteñas - dirigidas por afrouruguayos o hijos de uruguayos- que tuvieron una presencia sostenida en el tiempo y llegaron a ocupar - ahora regularmente- el espacio público de la ciudad con ensayos semanales en plazas o desfilando por las calles de San Telmo. Con el tiempo se fueron desprendiendo y/o conformando otras comparsas ${ }^{13}$, continuando la progresiva expansión -descentralización- del candombe fuera del histórico barrio de San Telmo hacia otras partes de la ciudad ${ }^{14}$. La llegada del corriente siglo también trajo la realización de las cada vez mayores Llamadas anuales - Llamadas en las que desfilan comparsas distintivas, en continuidad con el Desfile de Llamadas del carnaval montevideano- ${ }^{15}$. Estos desfiles fueron organizados primero con el auspicio de instituciones oficiales de promoción social o cultural del barrio - las Llamadas de Avenida de Mayo- y luego con el del gobierno municipal de turno - las Llamadas de San Telmo-, y su realización atrajo el interés de los medios de comunicación.

Sin embargo, por fuera de estos contextos a modo de espectáculo circunscrito en el calendario anual, el crecimiento meteórico del candombe y la ocupación del espacio público mediante esta práctica cultural se ha realizado plagada de conflictos con la policía, los vecinos y otros actores sociales (Frigerio y Lamborghini, 2009). Por ello, si bien el candombe afroargentino y el candombe afrouruguayo constituyen dos modalidades distintas, y a pesar de la distancia temporal entre la práctica en las calles de nuestra ciudad en uno y otro caso -últimas décadas del siglo XIX y del XX, respectivamente- ${ }^{16}$ me interesa

agrupaciones. Para un análisis en detalle de este proceso y de la expansión del candombe afrouruguayo en general, véase Frigerio y Lamborghini (2009). Por su parte, Parody (2014) focaliza en las acciones y narrativas de los candomberos de la primera generación, analizando su (in)migración no solo en términos socioeconómicos sino, principalmente, como exiliados de la dictadura uruguaya — que también lidiaron con la represión de la dictadura argentina-

13 En Uruguay, las Sociedades de Negros y Lubolos que actúan en el carnaval montevideano se denominan más comúnmente comparsas (Arce Asenjo, 2008, p. 129). En Buenos Aires - y en Argentina en general一, el término comparsa refiere a una agrupación de tamborileros/as y bailarinas/es con carácter menos formal que en el caso uruguayo que logra un cierto grado de autoidentificación y permanencia en el tiempo; este grado de permanencia está relacionado también con la permanencia en el lugar de ensayo y reunión. Como veremos, en las agrupaciones estudiadas la integración de los miembros se da principalmente por vínculos afectivos de amistad y tienen menor peso o presencia los vínculos familiares, como sí sucede en Uruguay y en las comparsas más cercanas al modelo uruguayo que aquí se desarrollan.

14 La formación de comparsas por fuera de San Telmo y la territorialización de otros barrios no excluyen la intensificación de la apropiación candombera de este primer barrio, sobre todo en los últimos dos años.

15 El Desfile de Llamadas del carnaval montevideano se lleva a cabo - en general - un viernes entre febrero y marzo de cada año. Las Sociedades de Negros y Lubolos recorren la calle Isla de Flores y su actuación es evaluada por un jurado (Arce Asenjo, 2008, p. 129).

16 Aquí enfoco la práctica del candombe en tanto grupos de tamboreo cuya performance se desarrolla en la calle. La 
plantear una línea de continuidad analítica en torno a los conflictos/ obstáculos enfrentados en tanto manifestaciones culturales "negras" en el espacio público de Buenos Aires (Frigerio y Lamborghini, 2009), dada la persistencia - el núcleo duro- (Lacarrieu, 2007) de la imagen de la ciudad "blanca", "europea", "moderna", que debe estar ordenada, tranquila, y en la cual es la cultura alta $-\mathrm{y}$ no la popular- la que tiene un lugar natural. Un contrapunto entre las prohibiciones pasadas y presentes del candombe en la ciudad de Buenos Aires revela la continuidad de prácticas regulatorias y disciplinadoras en tanto expresión del poder de la "blanquedad" como narrativa dominante de la ciudad-nación (Frigerio, 2006).

\section{Apropiaciones y resignificaciones del candombe afrouruguayo más allá de identidades negras}

En un tiempo relativamente corto, especialmente a partir de la década de 2000, el candombe afrouruguayo se difundió fuera del grupo migrante y pasó a ser practicado y apropiado cada vez más por distintos segmentos sociales de Buenos Aires.

Desde el año 2005, aproximadamente, aparecieron nuevos grupos compuestos mayormente por candomberos que pasaron por alguna de las agrupaciones pertenecientes a la primera generación de comparsas porteñas, y a partir de ese momento se intensificó la expansión de esta práctica cultural en sectores juveniles porteños. Jóvenes que aprendieron con $-\mathrm{y}$ participaron en- las primeras comparsas organizadas a comienzos de la década - lideradas mayormente por afrouruguayos - se desprendieron de las mismas y comenzaron a integrar o a organizar nuevos grupos. También fueron buscando conocimiento en maestros residentes en Montevideo - tanto a través de viajes a esa ciudad, como convocándolos o asistiendo a sus talleres en Buenos Aires-. Algunos de ellos empezaron a realizar tareas de docencia brindando talleres de candombe y sus alumnos generalmente

revisibilización del candombe argentino en las últimas décadas (Cirio, 2007; Frigerio y Lamborghini, 2011a) corresponde mayormente a la actuación de grupos de escenario. 
se fueron integrando al entonces ampliado circuito candombero. Claro está, estos trayectos han sido y son recurrentes, pero no exclusivos, otros se acercaron y acercan a la práctica de acuerdo a sus propios recorridos como músicos/bailarinas ${ }^{17}$, entre otros caminos posibles ${ }^{18}$.

A continuación, plantearé algunos temas relativos a los procesos de relocalización, apropiación y resignificación del candombe en manos de estos nuevos sectores juveniles ${ }^{19}$. Referirme a desarrollos novedosos del candombe local que tienen fuerte protagonismo de jóvenes porteños blancos de sectores medios ${ }^{20}$ implica una clasificación que responde a la dimensión relacional del campo candombero y a uno de mis supuestos de base: las distintas variables étnico-raciales, nacionales, generacionales y de clase que atraviesan a los actores sociales que practican candombe en la ciudad tienen incidencia en la configuración de sus acciones, representaciones e identificaciones candomberas - $\mathrm{o}$, más ampliamente, en las distintas construcciones del candombe-. De manera general, los y las candomberos/as en los que centro mi atención realizan un aprendizaje del candombe muy distinto al que sucede dentro de la comunidad de origen afrouruguaya donde, de modo similar a lo que ocurre con la incorporación de otros saberes performáticos de matriz africana, determinados principios o patrones generadores de comportamiento o de prácticas culturales,

17 Dentro de este contexto de expansión, en los últimos años la danza del candombe ha sido aprendida y apropiada por numerosas mujeres (Padilla y Lamborghini, 2013). Bajo el término de 'candomberos/as' englobo indistintamente a tamborileras/os y bailarinas.

18 Actualmente, existe cerca de una quincena de comparsas locales — contando solamente las del área metropolitanaque ensayan en plazas y lugares públicos. El candombe también tiene una fuerte presencia en el conurbano y en la provincia de Buenos Aires, así como en las principales capitales y en multiplicidad de ciudades y localidades a lo largo del territorio nacional. Dentro de la bibliografia específica sobre el candombe afrouruguayo en Argentina (Broguet, Picech y Rodríguez, 2013; Domínguez, 2009; Espinosa, 2013; Frigerio y Lamborghini, 2009 y 2012; López, 2002; Parody, 2014), la reconstrucción del surgimiento y desarrollo de las experiencias candomberas analizadas ha sido fruto de mi propia investigación.

19 Aunque debido a la franja etaria a la que pertenecen los sujetos del estudio —entre 25 y 35 en promedio—, sería más exacta su clasificación como adultos-jóvenes, utilizo el término 'jóvenes' como categoría analítica partiendo de la heterogeneidad de actores sociales que abarca y en tanto no corresponde a un grupo social determinado ni significa algo en sí, de modo a-temporal y a-histórico. Por el contrario, la categoría de 'jóvenes/juventud' cobra significados de acuerdo a su dimensión relacional y contextual (Chaves, 2010; Reguillo, 2000/2012). La noción de 'generación' alude a la socialización histórica de los sujetos y excede, como la de lo juvenil, su edad cronológica.

20 Los grupos observados están mayormente conformados por candomberos en los que se distingue la simultaneidad o interseccionalidad de los diferentes clivajes implicados en esta clasificación. En este punto, me interesa destacar que el factor nacional está aquí expresado bajo la categoría de 'porteños', pues es en Buenos Aires donde he realizado un trabajo de campo extendido. Si bien una referencia más directa a las expresiones del candombe en contextos provinciales excede los marcos de este trabajo, es importante notar que varias de las características de las nuevas construcciones del candombe que abordaré son compartidas y/o afines entre sectores juveniles de Buenos Aires y de otras ciudades argentinas, teniendo en cada caso, claro está, recorridos e historias diversas. 
se transmiten generacionalmente en forma consciente o inconsciente a través de la participación repetida en performances grupales como parte de la memoria habitual (Frigerio, 2000, p. 33). Lejos de amedrentarse frente a su carencia de las credenciales étnico-raciales-nacionales asociadas a la práctica - lo que émicamente suele ser referido como el hecho de no haber "mamado" el candombe desde la cuna-, estos actores candomberos se afirman en las propias construcciones. La cita que transcribo a continuación ${ }^{21}-\mathrm{el}$ testimonio de un integrante masculino de comparsa de Buenos Aires dado el 29 de octubre de 2011- da cuenta de la práctica del candombe en tanto no ligada a una socialización ni entorno cultural primarios, pero no por ello menos vigorosa, y cuyas formas pueden trascender siendo semilla de generaciones futuras:

Sí, yo lo que pensaba es que arrancó de mí. Yo no aprendí candombe, a mí no me enseñó un uruguayo a tocar candombe, ¿no?... Yo registro ese momento ahora. Estamos como en una etapa de transmisión en donde hay que dar cuenta del recorrido de nuestros ancestros, de los que vienen por detrás, pero... Algo que me pareció muy interesante es poder tomar conciencia de que nosotros estamos en una época, por lo menos con el candombe, como movimiento que nos convoca... Pensando en las generaciones que vienen podría pensarse, ¿no?... Estamos siendo semilla de lo que viene por delante. Yo no iba a escuchar candombe cuanto tenía 5 años.

Como hemos indicado, la difusión y las características que cobra el candombe entre estos sectores sociales responde no solo a factores de tipo interno relativos a su historia en Buenos Aires (la dinámica de las distintas camadas de afrouruguayos que lo transnacionalizaron; el fundamental proyecto de Kalakan-Güé; la dispersión y/o fragmentación de las comparsas, etc.), sino también a las transformaciones del campo cultural -y político- de Buenos Aires, relacionadas a su vez con la socialización generacional de estos candomberos/as. Teniendo en cuenta la imbricación de estos procesos en la reasignación de significados en torno a su adopción del candombe, afines a las propias experiencias vivenciales, en las siguientes páginas daré cuenta de la

21 El contexto de la cita es un encuentro realizado por el colectivo Los Tambores No Callan (LTNC). 
puesta en práctica en el candombe de algunos valores y/o formas organizativas contraculturales, contrahegemónicas, alternativas, que tienen continuidad respecto de formas de acción colectiva y asociatividad emergentes en décadas recientes. Me referiré a la horizontalidad, la autogestión y a la rearticulación de sentidos del candombe como expresión de resistencia y a cómo estas dimensiones se combinan con maneras de concebir - y apropiarse- del espacio público urbano ${ }^{22}$.

\section{Maneras alternativas de organización de los grupos de candombe: la horizontalidad}

Los nuevos actores sociales que se suman al candombe viven una experiencia colectiva comunalizante ${ }^{23}$ a la que le imprimen nuevas significaciones. El énfasis en la comunalización ya no pasaría por la (re) constitución de la comunidad afro o de determinados linajes familiares y lazos barriales como sucede en Montevideo (Ferreira, 199924), o en Buenos Aires con los inmigrantes afrouruguayos - y sus descendientes - (Parody, 2014), sino por el (re)conocimiento, el encuentro, el disfrute, entre jóvenes que comparten —en realidad, aprenden a compartir-gustos musicales y performáticos similares.

Estas diferencias implican reelaboraciones en las formas de organizarse -tanto al interior de las comparsas como en los eventos candomberos que convocan- que contrastan con las jerarquías que, en términos de senioridad, experiencia, capacidad performática y en ocasiones fenotipo vienen con el candombe más tradicional. Mientras que el principio de organización social/colectiva para la producción cultural/musical de los tambores en Uruguay - y de las comparsas

\footnotetext{
22 Aunque no lo desarrollaré en esta oportunidad, me interesa señalar que, dentro de estos procesos recientes y novedosos, se encuentra también la subversión de los límites de género construidos alrededor de la asociación tradicional hombretambor. Esto puede verse no solo en la formación de cuerdas femeninas (comparsas de mujeres) — desde 2007 en la capital y como fenómeno creciente de distintas ciudades del país en años posteriores-, sino también en la reivindicación y puesta en práctica de relaciones igualitarias de género en construcciones mixtas.

23 Entiendo, con Brow (1990), la comunalización como "cualquier patrón de acciones que promueve un sentido de pertenencia conjunta" (p. 1)

${ }^{24}$ Ferreira (1999) destaca la función de la música de tambores como facilitadora de la construcción del tejido social a lo largo de las generaciones y de los procesos identificatorios - masculinidad, afrodescendencia, ser tambor y pertenecer a una sociedad de tambores y a un barrio montevideano-, enlazando la subjetividad y corporeidad de los sujetos al contexto sociohistórico uruguayo.
} 
porteñas cercanas a este modelo más jerárquico- se basa "en la senioridad de los músicos/tambores, principio que guía la formación espacial del grupo y el orden interactivo en la performance" (Ferreira, 1999, pp. 44-45), las nuevas construcciones candomberas a las que nos referimos esbozan maneras alternativas de manejo del grupo y ponen en práctica el valor de la horizontalidad. Mediante formas de organizarse y de transmisión del conocimiento performático propias, la estructura organizacional del candombe es reorientada hacia una vivencia de las relaciones colectivas en tanto comunalización horizontal. Principios como estos ponen en tensión el respeto por Montevideo como la cuna del candombe y la ruptura con un código candombero tradicionalista y verticalista. La manifestación más notable de esta característica diferencial la constituyen las experiencias de comparsas sin directores. Por un lado, esta forma de organizarse es ciertamente más propicia en agrupaciones en las que los lazos que se tejen son mayormente de amistad - la familia se construye, se elige- entre pares. Por otro, dialoga con un contexto generacional en el que se destaca la emergencia de nuevos movimientos sociales (Svampa, 2008, 2011) y de grupos culturales alternativos que tienen el principio de la horizontalidad como una de sus características notorias (Wortman, 2009).

En cuanto a la organización del grupo, la horizontalidad en el candombe no implica solo el hecho de constituirse como una comparsa sin la figura de un director; afirmarse en una manera no jerárquica es un principio alrededor del cual se tejen otros principios organizativos. Dado que estas nuevas formas de regulación de las relaciones sociales se aprenden - y no vienen dadas-, cuestiones como la toma de decisiones en torno a las proyecciones del año; los eventos en los que participar; la relación con el barrio y/o la comunidad más extensa, entre otras, plantean desafios que deben manejarse en el mismo proceso de construcción, como puede observarse en el siguiente testimonio de una integrante femenina de comparsa de Buenos Aires, dado el 27 de abril de 2012:

[...] Siento que cuando uno elige este tipo de grupos se trata de aprender y dejar los egos a un lado, no es lo mismo permitir hablar que escuchar, yo si quiero puedo permitir hablar a 20 y escucho a 
uno nada más. Estamos en el proceso de que los que no se animaban a hablar, hablen, y de los que siempre tuvimos la voz, callemos y empecemos a escuchar. Es un proceso complicado. Se juegan cuestiones de confianza, de compañerismo, el buscar lo común en la diversidad, porque somos rediferentes en la comparsa a nivel de lo que queremos. [...] Son construcciones recomplicadas y estamos queriendo hacer algo que va en contraposición a lo que vivimos cotidianamente. En ningún lado te ceden la palabra, es más, te pisotean. Hablo en el común de cómo vivimos. Intentamos que ese espacio tenga otras características y es dificil.

Sin embargo, como también puede observarse en la cita precedente, estos desafios; la vivencia de lo "complicado" de este tipo de construcciones colectivas, son al mismo tiempo valorados como modos de sociabilidad alternativos - contrapuestos- a lo que ofrece la vida cotidiana.

\section{El valor de la autogestión en la organización de eventos candomberos}

La horizontalidad - y también el disfrute/alegría, el encontrarse y la no competencia- que se presentan como los valores principales que guían la práctica en las relaciones intra e intergrupales, se conjugan con otros principios relevantes para la organización de eventos candomberos - aglutinadores de los distintos grupos-. Entre estos, probablemente el que más se destaca es el de autogestión y/o independencia - en tanto carácter de independiente- En diálogo con formas de asociatividad emergentes post 2001, en el que este principio se pone de relieve en numerosas organizaciones sociales y, particularmente, en una variedad de proyectos culturales (Wortman, 2009), desde hace varios años la autogestión se implementa en la organización de una Llamada de candombe - anual y por comparsas-, evento que por excelencia convoca, aglutina y relaciona a los distintos grupos entre sí y con un público receptor y acompañante. La Llamada de candombe independiente Lindo Quilombo surgió en 2009 de una necesidad/interés de autogestión en esta actividad, y por varios años 
se diferenció de la Llamada de San Telmo — que informalmente pasó a denominarse oficial-, apoyada por el gobierno de la ciudad ${ }^{25}$. La construcción de la identidad de la Llamada Lindo Quilombo - y del colectivo Lindo Quilombo (LQ) que la organiza- ha girado principalmente en torno a lo independiente y autogestivo de su realización, conjugándose en este caso dos vectores: el repudio a las políticas culturales y sociales de la administración del actual gobierno de la ciudad -en, y más allá de las Llamadas de candombe- y la autogestión como principio de organización social en sí.

En términos organizativos, la autogestión de una Llamada de esta envergadura - con un promedio de veinticinco comparsas participantes-implica una serie de requerimientos y tareas de distintos niveles de complejidad: difundir el evento; gestionar los autobuses para el traslado de las comparsas locales y de Montevideo; tramitar los permisos burocráticos para el desfile; ocuparse del corte de las calles -y conseguir la ayuda policial para esto-; conseguir baños químicos; asegurarse de un lugar para alojar a las comparsas y/o candomberos que viajan desde el interior y de Uruguay, así como para realizar el encuentro del día posterior a la Llamada - estos dos lugares suelen coincidir-; planificar la comida del fin de semana, etc. ${ }^{26}$.

Los organizadores de LQ ponen en marcha esta Llamada bajo los valores de la autogestión, a los que suman la no espectacularización y la no mercantilización del candombe, destacando la no-competencia y el carácter abierto de la Llamada sin condiciones ni requisitos de ningún tipo. Al cabo de varios años, e incluso con cambios significativos en la Llamada de San Telmo —que desde 2013 pasó a ser organizada por las comparsas con el apoyo del gobierno de la

25 Cabe aclarar que este apoyo dista mucho de las políticas estatales del carnaval montevideano. En Buenos Aires, el gobierno local ha destinado escasos recursos y atención, lo cual ha traído constantes conflictos - y también lecturassobre las Llamadas de San Telmo. Un desarrollo de la historia de esta Llamada independiente desde su gestación hasta la actualidad excede los propósitos y espacio de este artículo. Por lo pronto, y más allá de los cambios, las disputas y consensos logrados en este camino, cabe destacar la permanencia y el trabajo exhaustivo de un núcleo de candomberos/ as protagonistas de las nuevas construcciones del candombe de las que doy cuenta de manera general.

26 Para la recaudación de los fondos necesarios, los integrantes de LQ organizan fiestas con música en vivo, venden remeras, calcomanías y bonos estampados con el logo de la Llamada. Así mismo, desde su segunda edición en 2010, LQ ha conseguido ciertos apoyos externos, algunos de los cuales se han mantenido y fortalecido con el transcurso de los años y que han facilitado locales para reunirse, autobuses y difusión. Se trata de sindicatos contestatarios y de organizaciones sociales interesadas en apoyar iniciativas en torno a la cultura popular y cuyo vínculo con LQ ha surgido de contactos personales y no de presentaciones formales, como tampoco de relaciones más o menos institucionalizadas. 
nación y no de la ciudad-, la Llamada independiente se mantiene como uno de los eventos principales del año, en base al camino realizado y al mayor afianzamiento de una identidad hacia adentro y de su proyección hacia afuera.

Por lo demás, y más allá de que mi foco esté puesto en Buenos Aires, es importante notar que, mientras que la modalidad autogestiva en la organización de una Llamada independiente por parte del colectivo Lindo Quilombo se manifiesta como apropiación del espacio público mediante una Llamada de candombe sin intermediarios estatales, este eje organizativo conlleva una dimensión menos politizada - pero no por ello carente de significados políticos en sentido amplio- en los denominados Encuentros de CandombeS, realizados desde 2007 en distintas ciudades y aglutinadores de comparsas de todo el país -y en ocasiones también del Uruguay- ${ }^{27}$. El mismo principio rige para otro tipo de encuentros candomberos realizados en la capital y otras ciudades argentinas.

\section{Formas de interfase entre cultura y politica: apropiaciones del candombe como "resistencia"}

Otra de las resignificaciones más relevantes observadas es la idea y vivencia de la "resistencia" — social, cultural, política- como expresión del candombe. Es sabida la vinculación entre la noción de resistencia y las prácticas de cultura popular negras (Hall, 1998/2003). En cuanto al candombe como expresión cultural más notoria de los afrodescendientes en Uruguay, Ferreira $(1999,2008)$ se refiere a la conformación de un ethos guerrero que aparece ligado tanto al carácter de la performance como en su relación con la sociedad envolvente. Respecto de esta última relación, la conducta guerrera que supone la marcha de los tambores, nos dice el autor (Ferreira, 1999, pp. 118-119) ${ }^{28}$, refiere al hecho de que los tambores:

27 Los denominados Encuentros de CandombeS se iniciaron en 2007 en la ciudad de Córdoba —con antecedentes en encuentros de La Plata- y al cabo de unos años empezaron a rotar por distintas ciudades y localidades del territorio nacional.

28 En un trabajo posterior, el autor explicita aun más la relación entre el candombe y el concepto de resistencia, al plantear la cultura performática afrouruguaya como la "constitución de un dominio cultural alternativo al dominante. 
Re-apropiándose literalmente del espacio, históricamente han burlado la dominación y la vigilancia del Estado en la circulación y las relaciones interpersonales, afirmando lo urbano como lugar público. Al presente, el colectivo organizado logra en su movimiento y su penetración acústica en un entorno sonoro de muchas cuadras transversales, una re-territorialización de un espacio geográfico en la urbe con un sentido de cultura negra.

Ahora bien, ¿de qué tipo de resistencia estariamos hablando cuando las expresiones culturales afroamericanas como el candombe son corporizadas y se imbrican con las subjetividades de sectores medios "blancos" no-subalternizados? En las resignificaciones llevadas a cabo por los nuevos actores sociales en el candombe analizados, la resistencia en/del candombe puede aparecer asociada a la subalternidad-resistencia de los sectores populares negros. Esta apelación no se refiere solo a los afrodescendientes en Uruguay, sino que incluye reflexiones sobre procesos históricos de los cuales Argentina no es excluida, ya que, como advierte Domínguez (2009, las narrativas sobre la ascendencia europea de la cultura nacional y su racialización como blanca "no eliminaron la presencia de lo negro o africano en los debates sobre lo argentino" (p. 91). Por el contrario, “contribuirán con la elaboración de un mapa en el cual los negros y sus expresiones culturales ocupan el lugar de la alteridad y en algunos casos de lo subalterno" (p. 91). Así, fruto de la lectura opositora que realizan estos sectores juveniles de la narrativa dominante de la nación "blanca-europea", la resistencia histórica de los esclavizados y sus descendientes a la esclavitud y a la cultura hegemónica es tomada para resignificar la práctica con un sentido politico amplio. Ante la frecuente hostilidad contemporánea hacia el género por parte de diversos actores sociales, tanto la animadversión como la resistencia a la misma, son frecuentemente insertadas dentro de una prolongada serie de contiendas cuyo origen puede establecerse en la época de la esclavitud.

De esta manera, aunque los sentidos politicos asignados al candombe no aparecen en estos casos vinculados a reclamos para la

La performance aparece como experiencia que se organiza más allá del control social dominante y donde el propio cuerpo deviene un sitio de resistencia" (Ferreira, 2008, p. 116). 
cultura afro o para los afrodescendientes, los conceptos de 'resistencia' y de 'lucha' se apoyan en su significante negro (Hall, 1998/2003). En un nivel explícito, estas resignificaciones aparecen cristalizadas en el caso de la Llamada de candombe independiente analizada previamente. El esfuerzo en pos de una presencia autónoma del candombe -bajo la forma de una Llamada anual- en el espacio público es particularmente importante en momentos en que un gobierno de derecha controla la ciudad de Buenos Aires. La independencia respecto de dicho apoyo institucional implica llevar a la práctica un alineamiento ideológico acometedor respecto de lo que se considera que este arte se merece y, entonces, lo autogestivo de la Llamada independiente se resignifica como resistencia ${ }^{29}$.

Así mismo, como he indicado a fin de complejizar la idea de apropiación en un sentido creativo - y no como expropiación-, para estas rearticulaciones de sentido en que se conjugan apropiación y resistencia es necesario ir - también- más allá del campo de estudio de la/s cultura/s negra/s. Entonces, podemos remitirnos a la conocida asociación entre el concepto de resistencia y el campo de la música popular en general, punto en el que Alabarces (2008) indica que un concepto ampliado de subalternidad permite extender la noción de resistencia y que, desde este enfoque, "las músicas populares han sido pensadas más de una vez como espacios simbólicos de resistencia político-cultural" (p. 2). Reguillo (2000/2012) por su parte se refiere a los procesos de apropiación y resistencia en el campo de las culturas populares y los compara con las formas que estos toman en el campo de las culturas juveniles. Una particularidad de nuestro caso tiene que ver con dimensiones de una apropiación juvenil de cultura popular; no se trata ni de la apropiación de objetos de la cultura dominante, ni de cultura globalizada y se llevan a cabo prácticas - los sujetos producen cultura- y no consumos. Algunas de las resignificaciones del candombe en tanto cultura de resistencia articulan con lo popular

\footnotetext{
29 A su vez, esta resistencia se ancla en una memoria negra local. Esto es notable en la apropiación del término 'quilombo', resemantizándose una palabra de origen bantú que denominó los primeros focos de resistencia de los africanos al esclavismo colonial, que en la actualidad tiene una dimensión importante en la lucha de los afrodescendientes (Boaventura, 2000, p. 336) y que, por contraste, en la jerga coloquial local conlleva aspectos negativos. En el caso de los organizadores de la Llamada independiente Lindo Quilombo, este anclaje también se aprecia en la elección del barrio - primero San Telmo, luego Montserrat - y en la fecha de la Llamada — cercana al 20 de noviembre, día de la conciencia negra en Brasil一.
} 
- significado como la alegria, lo colectivo, la expresión del cuerpo en el espacio público - de una práctica cultural además racializada, es decir; vinculada a sectores subalternos racializados. En lo colectivo, la práctica consciente de este arte conlleva una sociabilidad y solidaridad valoradas como formas que ayudan a trascender ciertos aspectos de la vida moderna con sus rasgos de individualismo y consumo. Además, a la comunalización que propone la organización social del candombe $^{30}$, estos sectores candomberos superponen una propuesta adicional de comunalización horizontal, alternativa a los modos jerárquicos de organización más tradicionales.

Como anticipé, otra dimensión en torno a la resistencia es la que pone de relieve al candombe como manifestación cultural popular que se produce en el espacio público de la ciudad -más aun cuando su práctica en el espacio público enfrenta frecuentemente adversidades-. Así, la ocupación regular del espacio público con los ensayos semanales de las comparsas puede conllevar una cualidad política en general, en la que se destaca la apropiación y práctica del espacio público mediante el candombe, a través de la alegria y de la fuerza de la expresión del cuerpo y de los tambores colectivamente. Este eje está en consonancia, a su vez, con la emergencia de nuevos ethos militantes (Svampa, 2008, 2011) ligados a la noción del activismo cultural que tienen como particular campo de acción y visibilidad el espacio público ${ }^{31}$.

\section{Consideraciones finales}

Desde que comenzó a consolidarse en la década de 1990, el campo de los estudios afrolatinoamericanos (Wade, 2006) ha crecido y au-

30 En la música de tambores afrouruguaya, la estructura altamente corporativa y basada en la comunicación-diálogo entre las distintas voces tambores) excede la performance musical y se imbrica con la organización social de la orquesta de candombe (Ferreira, 1999)

31 Por mi parte, me interesa poner en juego este concepto de 'nuevo ethos militante' (Svampa, 2008, 2011) con el de 'ethos guerrero' en el candombe afrouruguayo (Ferreira, 1999, 2008), para hablar de nuevos ethos militantes candomberos. Esta noción resulta particularmente relevante para desarrollos como el de Los Tambores No Callan (LTNC), colectivo candombero de militancia política — no partidaria— que desde 2008 visibiliza con candombe luchas y causas sociales, cuyo análisis excede los límites de este trabajo. 
mentado su riqueza en la variedad y complejidad de temas vinculados a las experiencias, representaciones y prácticas asociadas a la afrodescendencia, situados en contextos localizados y procesos históricos particulares (Guzmán y Geler, 2013). Dentro de los retos del camino por recorrer (Restrepo, 2013), me interesa subrayar la escasa atención que han recibido los procesos cada vez más extendidos en los que distintas manifestaciones de "cultura negra" se difunden para ser practicadas entre diversos sectores sociales y las formas en las que son significadas, dados los flujos globales y los contextos de promoción de lo multiétnico y multicultural (Sansone, 2003). En Argentina - como en otros contextos nacionales- un creciente número de jóvenes socialmente "blancos" se identifican, apropian y disfrutan de "culturas negras" - muchas de ellas relocalizadas-. Quedan pendientes -másanálisis que vayan más allá de los términos de consumo exoticista -y racializante-, o de los efectos más o menos nocivos de la "negrofilia blanca" (Sansone, 2003, pp. 110 y 211) en relación a estos fenómenos. Mientras que lo afro - y su estudio- adquieren cada vez mayor vigencia y complejidad en Latinoamérica, es necesario profundizar la reflexión sobre la disociación entre cultura, comunidad e identidad negras (Frigerio, 2000; Sansone, 2003) ${ }^{32}$.

De manera general y sintética, he propuesto algunas reflexiones teóricas sobre lo que tiene para decir la creciente difusión y práctica del candombe en su modalidad afrouruguaya de la sociedad argentina contemporánea o, para ser más precisa, sobre ciertos desarrollos que tienen lugar en la misma. En este trabajo sostuve que algunas construcciones de la movida candombera local protagonizadas mayormente por sectores juveniles medios socialmente blancos se intersectan con una escena cultural cada vez más diversificada y con las transformaciones sociales, culturales y políticas derivadas en gran parte de la crisis de 2001.

\footnotetext{
32 A los trabajos de Frigerio y de Sansone citados, que puntualizan en este problema teórico, agrego análisis de casos específicos en Argentina como los de Corti (2011) y de Domínguez (2009) - y mi propio trabajo (Lamborghini, 2015) - . Respecto del candombe afrouruguayo en Uruguay, Andrews (2007) trabaja temáticas relacionadas con este problema desde una perspectiva histórica, y Ferreira (1999) lo hace al analizar conyugalidades interraciales en contextos afromontevideanos de vecindarios multirraciales.
} 
En su encuentro con el candombe, la experiencia y significación de las características sociomusicales de la performance por parte de estos jóvenes, responden a negociaciones de sentido construidos - principalmente- alrededor de: 1) lo colectivo sobre lo individual; 2) lo público sobre lo privado; 3) el goce y el disfrute sobre la vida acartonada, de apariencias y consumo. He indicado cómo estos sectores sociales nuevos en el candombe lo corporizan como una forma de expresión que se opone/que se plantea alternativa a situaciones mayoritarias de distinto tipo, de una manera en que resuena lo señalado por Ferreira (1999, pp. 115) respecto del logro por y en la performance de un espacio y tiempo alterno. Claro que el sentido es distinto porque Ferreira (1999) apunta a la subalternidad y al capital cultural de los sectores populares negros uruguayos. En relación con estas rearticulaciones de sentido, hablé por ello de apropiaciones en tanto interpretaciones creativas y resignificaciones más allá de identidades y de grupos socialmente negros. Estos candomberos y candomberas se posicionan como una manera de trascender el problema de las pertenencias que en principio los sitúan como ajenos o en discontinuidad con la práctica - a la no pertenencia racial y nacional se suma la ajenidad que representa lo afro en nuestra cultura nacional supuestamente "blanca"- De manera general, lo hacen mostrando una fuerte continuidad con el carácter social o las funciones sociales señaladas por Frigerio (1992/2000) como una de las características destacables de las manifestaciones artísticas afroamericanas, las cuales "no sólo tienen una función generadora de communitas (en el sentido de Turner, 1969), sino que también permiten que el grupo como un todo (o sus distintos miembros) realicen comentarios sociales acerca de los temas que les atañen y que se puedan expresar, llegado el caso, los conflictos internos de la comunidad" (Frigerio, 1992/2000, p. 160). Con respecto al candombe en particular, la comunalización de las producciones culturales analizadas están en sintonía con la función integrativa y con la enorme vigencia de su capacidad de construcción de identidades postuladas por Ferreira $(1999,2001$, p. 55).

Por otro lado, también he reseñado las principales características que evidencian cambios que pueden incluso representar rupturas respecto de formas locales más tradicionales de candombe, las cuales, en 
mayor o menor medida, siguen pautas relacionadas con el desarrollo histórico en su contexto de origen del otro lado del Plata. Dado que los sentidos otorgados se encuentran más a tono con su experiencia vivencial, su socialización generacional $\mathrm{y}$, más específicamente, con determinadas construcciones culturales de su época, planteo que estas nuevas construcciones del candombe revelan su cualidad de vértice de varias intersecciones temáticas, mostrando su conexión con problemas que exceden la práctica del candombe y/o el despliegue de manifestaciones culturales afroamericanas. Así, la bibliografia relativa a las culturas juveniles y los nuevos movimientos sociales me orientó respecto de la promoción de nuevas formas de sociabilidad (Goldman, 2007 , p. 10) y sobre las maneras alternativas de organización (Reguillo, 2000/2012, p. 56) en el contexto post 2001 (Svampa, 2008, 2011; Wortman, 2009, 2010) como marco para apreciar las formas de relacionarse y de organización al interior de los grupos, siendo la horizontalidad el camino para consolidar lo colectivo por sobre lo individual de la figura del director de comparsa o de la jefatura de los mayores. Por eso hablé de una comunalización horizontal entre amigos-pares y de familias que se construyen/eligen a partir de lazos basados en el disfrute/alegria, el encontrarse y la no competencia, como valores principales que guian las relaciones intra e intergrupales. Como parte del mismo contexto, a partir del caso de la Llamada de candombe independiente Lindo Quilombo, trabajé también sobre el sentido de la autogestión y aludí a la no espectacularización y la no mercantilización de los eventos candomberos aglutinadores puestos en marcha.

Otra de las rearticulaciones de sentido destacadas se refiere al candombe como expresión de resistencia. A veces con consignas definidas, otras basándose simplemente en la idea de ocupar la calle -apropiarse, a menudo conflictivamente, del espacio público-, estos candomberos se articulan con lo popular del candombe - la alegría, lo colectivo, el uso del cuerpo en el espacio público-. Bajo esta lógica, la noción de resistencia que viene con la práctica - más tradicional- (Ferreira, 2008) y el posicionamiento crítico frente a la peculiar alterización de las otredades étnico-raciales en Argentina - su negación- confluyen en acciones variadas como el -mero- sentido político de ocupar la calle o la más explícita oposición al gobierno — de derecha— de la ciudad. 
En los casos en los que se explicita, aparece además la asociación de estas resistencias contemporáneas a la subalternidad -y resistencia- de los esclavizados frente a la esclavitud, y al candombe como performance política que posibilita la conciencia de la fuerza colectiva de quienes lo corporizan. Esto cobra una dimensión particularmente contestataria si se tiene en cuenta que el candombe marca el espacio público de Buenos Aires - una ciudad pensada y practicada hegemónicamente como "blanca" - imprimiéndole la fuerza del sonido de los tambores y de su danza.

Desde hace más de tres décadas, el espacio público de la ciudad de Buenos Aires - y crecientemente de otras ciudades del país- es apropiado y transformado con la potencia y la musicalidad, la fuerza rítmica, la expresividad corporal y lo festivo del candombe afrouruguayo. En este trabajo reflexioné acerca de prácticas y significados que dan forma a nuevas experiencias respecto de un universo del candombe más ligado a sus orígenes étnico-nacionales. Mostré cómo, a través de esta práctica cultural, muchos jóvenes se identifican y se posicionan como sujetos en, pero también más allá del ámbito del candombe, siendo esta performance colectiva una de las opciones salientes que eligen para relacionarse con sus pares y para proponer formas de articularse con la sociedad mayor. El candombe se constituye entonces como una alternativa para construir novedosos modos de sociabilidad, lucha política y apropiación/construcción del espacio público. Mediante esta práctica cultural, los sectores juveniles analizados se posicionan como actores sociales que dialogan - muchas veces en tensión- no solo con las formas tradicionales de hacer y concebir el candombe - tanto en Montevideo como, principalmente, en Buenos Aires-, sino también, crecientemente, con las formas tradicionales de entender la participación política y la ciudadanía.

\section{Referencias}

Alabarces, P. (2008). Posludio: música popular, identidad, resistencia y tanto ruido (para tan poca furia). Revista Transcultural de Música, 12. 
Andrews, G. R. (2007). Recordando África al inventar Uruguay: sociedades de negros en el Carnaval de Montevideo, 1865-1930. Revista de Estudios Sociales, 26, 86-104.

Arce Asenjo, D. (2008). El desfile de Llamadas como "ritual conmemorativo". En G. Goldman (Comp.), Cultura y sociedad afro-rioplatense (pp. 127-150). Montevideo: Perro Andaluz Ediciones.

Boaventura, L. I. (2000). Os quilombos no Brasil: questões conceituais e normativas. Etnográfica, IV(2), 333-354.

Bourdieu, P. (2011). El sentido social del gusto. Elementos para una sociología de la cultura. Buenos Aires: Siglo XXI Editores.

Briones, C. (2005). Formaciones de alteridad: contextos globales, procesos nacionales y provinciales. En C. Briones (Comp.), Cartografias argentinas: politicas indigenistas y formaciones provinciales de alteridad (pp. 9-36). Buenos Aires: Antropofagia.

Broguet, J., Picech, M. C. y Rodríguez, M. (2013). Candomberío. Imaginando los candombes del Litoral. En M. Ghidoli y J. F. Martínez Peria (Comps.), Estudios afrolatinoamericanos: nuevos enfoques multidisciplinarios. Actas de las Terceras Jornadas del GEALA [E-Book] (pp. 655-666). Buenos Aires: Ediciones del Centro Cultural de la Cooperación Floreal Gorini.

Brow, J. (1990). Notes on Community, Hegemony, and the Uses of the Past. Anthropological Quarterly, 63(1), 1-17.

Carvalho, J. J. (2002). Las tradiciones musicales afroamericanas: de bienes comunitarios a fetiches transnacionales. Serie Antropología UnB, 320, 1-25.

Chaves, M. (2010). Jóvenes, territorios y complicidades. Una antropología de la juventud urbana. Buenos Aires: Espacio Editorial.

Cirio, P. (2007). De Eurindia a Bakongo. El viraje identitario argentino después de la asunción de nuestra raíz afro. Entremúsicas: música, investigación y 
docencia. Recuperado de http:/ / entremusicas.fi les.wordpress.com/2008/04/ de-euindia-a-bakongo.pdf

Corti, B. (2011). Lo "afro" en el jazz argentino. Identidades y alteridades en la música popular. (Tesis de Maestría). Facultad de Ciencias Sociales, Universidad de Buenos Aires, Buenos Aires.

Dominguez, M. E. (2009). Suena el Río. Entre tangos, milongas, murgas e candombes: músicos e gêneros rio platenses em Buenos Aires (Tese de Doutorado). Florianópolis, Universidade Federal de Santa Catarina.

Espinosa, M. C. (2013, marzo). Los “candombes" como Memorias Vivas: reflexiones en torno a africanidades en la provincia de Salta, Argentina. Revista de Humanidades Populares, 6, 41-51.

Ferreira Makl, L. (1999). Las Llamadas de tambores: comunidad e identidad de los afro-montevideanos (Tesis de Maestria). Universidade de Brasília, Brasília.

Ferreira Makl, L. (2001). La música afrouruguaya de tambores en la perspectiva cultural afro-atlántica. Anuario de Antropología Social y Cultural en Uruguay, 41-57.

Ferreira Makl, L. (2008). Dimensiones afrocéntricas en la cultura performática uruguaya. En G. Goldman (Comp.), Cultura y sociedad afro-rioplatense (pp. 91-123). Montevideo: Perro Andaluz.

Ferreira Makl, L. (2013). Desde el arte a la politica y viceversa en los ciclos de política racial. En F. Guzmán y L. Geler (Eds.), Cartografías afrolatinoamericanas. Perspectivas situadas para análisis transfronterizos (pp. 217-240). Buenos Aires: Biblos.

Frigerio, A. (2000). Cultura negra en el Cono Sur: representaciones en conflicto. Buenos Aires: EDUCA.

Frigerio, A. (2006). "Negros" y "Blancos" en Buenos Aires: repensando nuestras categorias raciales. Temas de Patrimonio Cultural, 16, 77-98. 
Frigerio, A. (2013). Umbanda and Batuque in the Southern Cone: Transnationalization as cross-border religious flow and as social field. En C. Rocha and M. Vasquez (Eds.), The diaspora of Brazilian religions (pp. 165-195). Boston: Brill.

Frigerio, A. y Lamborghini, E. (2009). El candombe (uruguayo) en Buenos Aires: (Proponiendo) Nuevos imaginarios urbanos en la ciudad "blanca". Cuadernos de Antropología Social, 30, 93-118.

Frigerio, A. y Lamborghini, E. (2011a). (De)Mostrando cultura: estrategias políticas y culturales de visibilización y reivindicación en el movimiento afroargentino. Boletin Americanista, 63, 101-120.

Frigerio, A. y Lamborghini, E. (2011b). Procesos de reafricanización en la sociedad argentina: umbanda, candombe y militancia "afro". Pós Ciencias Sociais, 16, 21-35.

Frigerio, A. y Lamborghini, E. (2012). Encontrarse, compartir, resistir: una "nueva construcción" del candombe (afro)uruguayo en Buenos Aires. Anuario de Antropología Social y Cultural en Uruguay, 10, 95-113.

Geler, L. N. (2011). ¿Quién no ha sido negro en su vida? Performances de negritud en el carnaval porteño de fin de siglo (XIX-XX). En P. García Jordán (Ed.), El Estado en América Latina. Recursos e imaginarios, siglos XIX-XXI. Barcelona: Universidad de Barcelona.

Goldman, M. (2007). Introducao: Politicas e Subjetividades nos "Novos Movimentos Culturais”. ILHA, 9(1-2), 8-23.

Guzmán, F. y Geler, L. (2013). Para unas cartografias afrolatinoamericanas. En F. Guzmán y L. Geler (Eds.), Cartografias afrolatinoamericanas. Perspectivas situadas para análisis transfronterizos (pp. 13-21). Buenos Aires: Biblos.

Hall, S. (1998/2003). Da diáspora: Identidades e mediações culturais [L. Sovik (Org.)]. Belo Horizonte: Editora UFMG.

Lacarrieu, M. (2007). La 'insoportable levedad' de lo urbano. Eure, XXXIII(99), 47-64. 
Lamborghini, E. (2015). Candombe afro-uruguayo en Buenos Aires: nuevas formas de sociabilidad, política y apropiación del espacio público (Tesis de doctorado). Universidad de Buenos Aires, Buenos Aires.

López, L. (2002). Candombe y negritud en Buenos Aires: una aproximación a través del folklore (Tesis de Licenciatura). Facultad de Filosofia y Letras, Universidad de Buenos Aires, Buenos Aires.

Padilla, M. y Lamborghini, E. (2013, agosto). La "bailarina" del candombe (afro-uruguayo): cuerpo, movimiento y transformación. Una mirada desde su práctica y enseñanza en un nuevo contexto social. Conferencia presentada en el III Congreso internacional de Artes en Cruce, Buenos Aires.

Parody, V. (2014, enero-junio). Música, política y etnicidad: convergencias entre democracia y dictadura en el proceso de relocalización del candombe afrouruguayo en Buenos Aires (1973-2013). Resonancias, 18(34), 127-153.

Reguillo, R. (2000/2012). Culturas juveniles. Formas Politicas del desencanto. Buenos Aires: Siglo XXI Editores.

Restrepo, E. (2013). Estudios afrolatinoamericanos: posibles aportes desde los estudios culturales. En F. Guzmán y L. Geler (Eds.), Cartografias afrolatinoamericanas. Perspectivas situadas para análisis transfronterizos. Buenos Aires: Biblos.

Rinaudo, C. (2010). Más allá de la "identidad negra": mestizaje y dinámicas raciales en la ciudad de Veracruz. En E. Cunin (Ed.), Mestizaje y diferencia. Politicas y culturas de "lo negro" alrededor del Caribe (pp. 225-266). México D.F: Instituto Nacional de Antropología e Historia.

Sansone, L. (2003). Blackness without Ethnicity: Constructing Race in Brasil. New York: Palgrave MacMillan.

Segato, R. L. (1998). Alteridades históricas/identidades políticas: una crítica a las certezas del pluralismo global. Série Antropologia, 234, 2-28. 
Sheriff, R. (1999). The theft of carnival: National spectacle and racial politics in Rio de Janeiro. Cultural Anthropology, 14(1), 3-28.

Svampa, M. (2008). Cambio de época. Movimientos sociales y poder politico. Buenos Aires: Siglo XXI Editores.

Svampa, M. (2011). La política en las calles: lenguajes de movilización y espacio público en la época contemporánea. En M. Z. Lobato (Ed.), Buenos Aires. Manifestaciones, fiestas y rituales en el siglo XX. Buenos Aires: Biblos.

Wade, P. (2006). Afro-Latin Studies: Reflections on the field. Latin American and Caribbean Ethnic Studies, 1(1), 105-124.

Wortman, A. (Comp.) (2009). Entre la politica y la gestión de la cultura y el arte: nuevos actores en la Argentina contemporánea. Buenos Aires: Eudeba.

Wortman, A. (2010). Cambios culturales, cambios en los consumos culturales. Revista Indicadores Culturales, 100-109.

\section{Cómo citar este artículo}

Lamborghini, E. (2017). Apropiaciones y resignificaciones de las "culturas negras": la práctica del candombe afrouruguayo en sectores juveniles blancos de Buenos Aires (Argentina). Universitas Humanística, 83, 303-330. http:// dx.doi.org/10.11144/Javeriana.uh83.arcn 